\title{
PENICILLIN IN INFECTIONS OF INFANCY
}

\author{
BY \\ EDWARD C. ROSS COUPER, M.D., F.R.F.P.S., D.P.H. \\ Deputy Medical Superintendent, Walton Hospital, Liverpool
}

The high incidence of parenteral infection in infancy, however its rôle in the production of morbidity and mortality rates is interpreted, cannot be doubted. Many statistics are available in the literature. One of the latest surveys, for example, is that by Alexander and Eiser (1944) who found evidence of parenteral infection in 88.6 per cent. of their cases, the bulk of this infection taking the form of upper respiratory infection and otitis media. The common organisms were haemolytic streptococci, pneumococci and staphylococcus aureus. Parenteral infection is even more deadly in the premature infant whose powers of resistance are feeble.' In] Walton Hospital, Liverpool, evidence of parenteral infection has been found in one-third of premature infants coming to post-mortem examination. When penicillin became avilable for civilian use it was thought that parenteral infection in infancy might be a good field for its use and immediate steps were taken to test this possibility. A gift of a small quantity of penicillin was obtained and seven cases were treated. These are included in the series about to be described (group, A, no. 1,3 and 4; group B, no. 1 and 2; and additional cases, no. 2 and 4). The hopeful results justified application to the Penicillin Committee (Civilian) of the Medical School of the University of Liverpool and the remainder of the cases were treated with penicillin so obtained.

Type of case. Twenty cases are described. This is a small number but this is a preliminary report and perhaps the novelty of the treatment will excuse this defect. Owing to the necessity for economy in the use of penicillin the cases have been selected as those most likely to benefit and they fall into two groups.

(A) Premature infants. (a) The premature infant which goes steadily down hill. This type is frequently found at post-mortem examination to have some parenteral infection; and (b) the premature infant which begins to have cyanotic attacks about ten days after birth, often a manifestation of bronchopneumonia.

(B) Mature Infants. In this group the cases of parenteral infection which presented themselves happened to be all cases of otitis media manifesting itself by diarrhoea, vomiting, loss of weight, varying degrees of dehydration and signs of inflammation in the tympanic membranes.

Various difficulties are encountered in investigating this type of case. First there was the difficulty of obtaining adequate controls. The clinical examination of infants is often difficult, especially in the case of premature infants, making comparable groups of cases difficult to obtain. When an apparently efficacious method of treatment is thought to have been found, it is difficult to withhold such treatment for the sake of obtaining a control group. In other words, when dealing with infants it is difficult to justify on humane grounds a fully controlled experiment.

Secondly, there was the difficulty in assessing the part played by penicillin in any improvement which occurs. The weight curve is a useful guide to improvement in an infant, but degrees of improvement can occur which are clinically manifest before gain in weight is observed. Assessing improvement is largely dependent upon clinical judgement.

Thirdly, the difficulty in obtaining bacteriological confirmation of suitability for penicillin therapy. Often this is impossible and the treatment assumes a rather empirical form which can only be justified by results. Faeces were examined in nineteen of the twenty cases with negative result in sixteen. Three grew B. Morgan 1. In one case, the premature infant which died, a specimen was not obtained.

Method of treatment. It was decided to administer the penicillin systemically and 25,000 units were given daily, both to premature and mature infants. This amount of penicillin was made up in bottles containing $8 \mathrm{c} . \mathrm{cm}$. of saline and was given in $1 \mathrm{c.cm}$. doses three-hourly by intramuscular injection into the buttocks, each bottle lasting for twenty-four hours. Dehydration was combated by the administration by mouth of glucose saline ( $\frac{1}{5}$ normal saline and 5 per cent. glucose). If necessary intravenous drip saline was given (Hartmann's Solution). 
It. N.T.

A 1

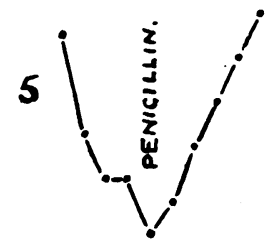

4 sepr. OCr.
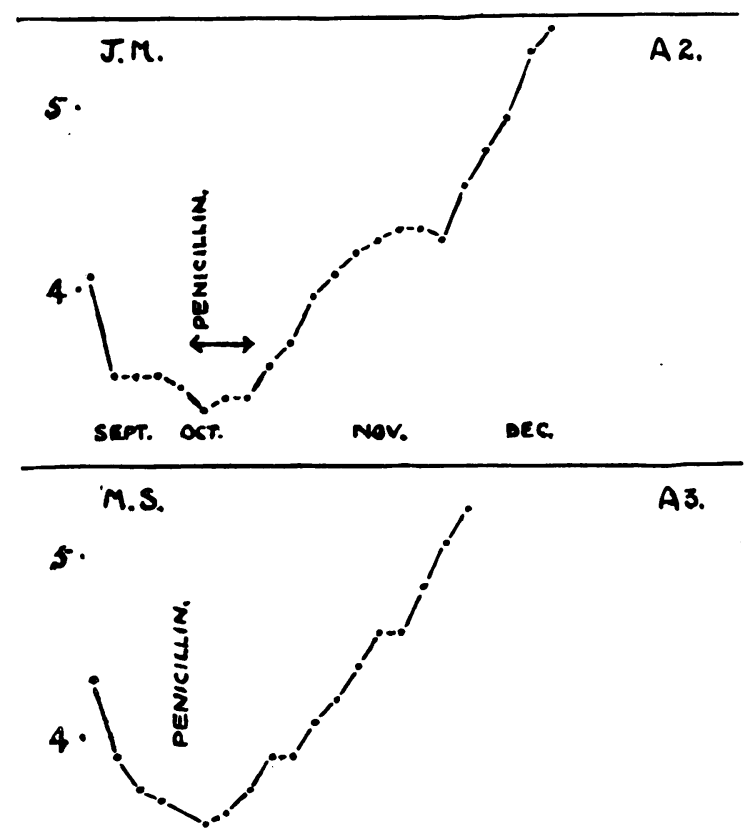

SEF.

OST.

Nov.

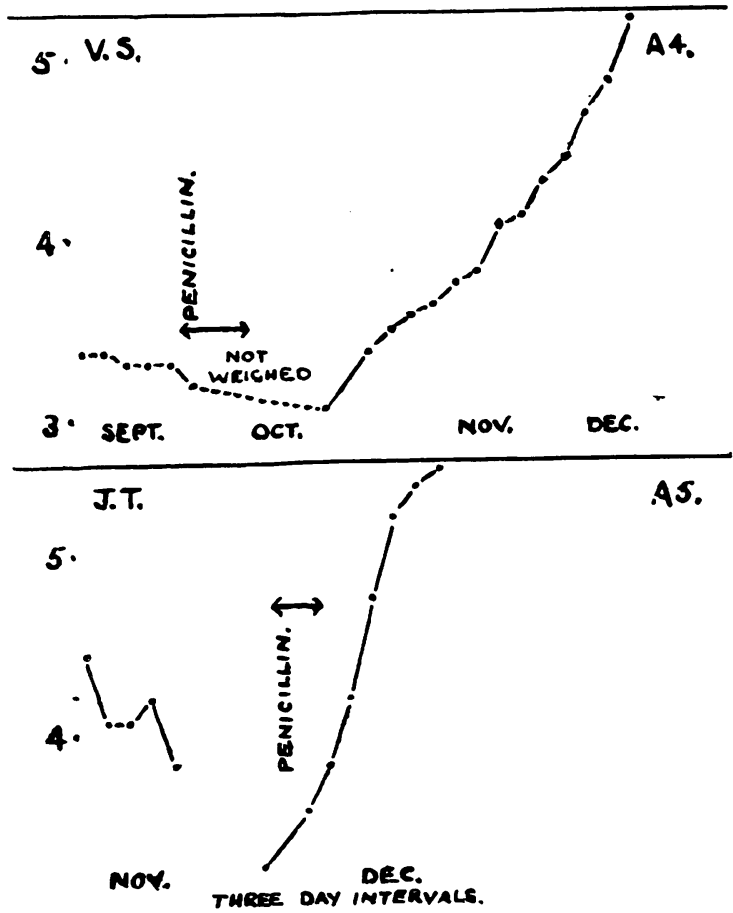

Description of cases

\section{Group (A).-Dremature infants}

1. N. T., male. Born 9-9-44. Premature infant which went steadily downhill from birth, developed mild thrush and some dehydration. No clinical evidence of infection. Penicillin treatment coincided with improvement. Total dose- 75,000 units. Later attended out-patients, weighing $7 \mathrm{lb} .9 \mathrm{oz}$.

2. J. M., male. Born 30-9-44. Premature infant with jaundice, which went steadily downhill. Penicillin treatment again coincided with clinical improvement and gain in weight. Total dose200,000 units.

3. M. S., male. Born 24-9-44. Premature infant with cyanotic attacks starting ten days after birth-probably bronchopneumonia. Reduction in number of attacks after penicillin. Very ill at the height of its attacks but apyrexial throughout. Total dose-100,000 units.

4. V. S., male. Born 19-9-44. Premature infant with cyanotic attacks beginning fourteen days after birth-probably bronchopneumonia. Penicillin was given and continued till attacks ceased, which took fifteen days. This infant was very ill indeed and was not weighed for some time. Total dose $-312,000$ units.

5. J. T., male. Born 4-11-44. Premature infant - probably bronchopneumonia, which was taken from hospital against advice and brought back nearly moribund. Gap in graph indicates period out of hospital. Penicillin coincided with improvement and gain in weight. Total dose-150,000 units. 
16. M.sh.

A6.

6 .
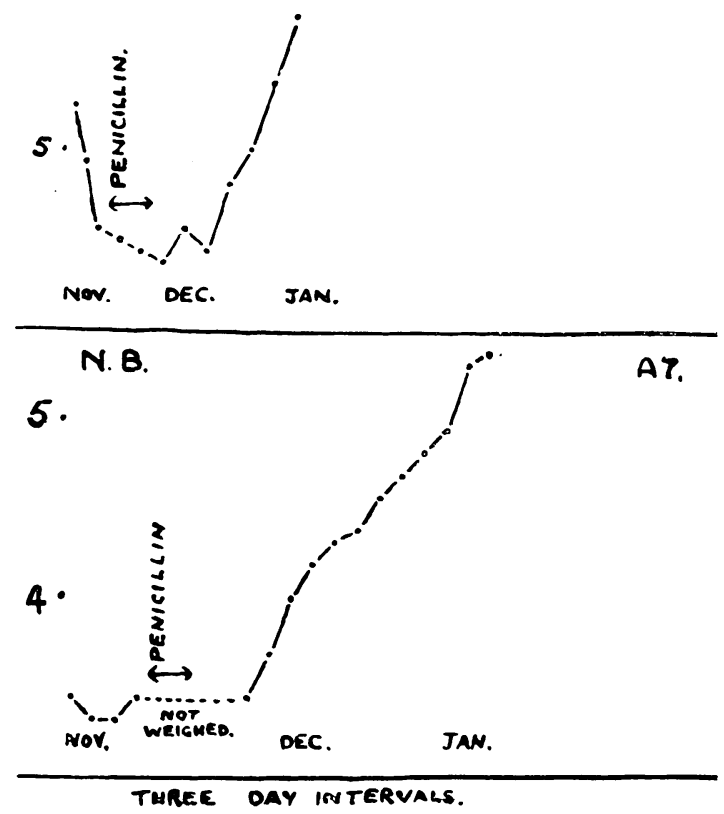

8. A. McK., female. Born 18-11-44. Weight at birth-3 lb. $14 \mathrm{oz}$. This premature infant was only weighed on four occasions, during which time its weight remained stationary. Ten days after birth several cyanotic attacks occurred. Respirations were laboured and alae nasi working. Penicillin was begun within four hours of the first cyanotic attack, but it only survived long enough to have three doses, i.e. 9375 units. Post-mortem examination revealed the presence of marked bronchopneumonia in both lungs, confirmed by section. Direct smear from the lung showed grampositive diplococci and large numbers of coliform bacilli. Culture showed coliform bacilli and nonhaemolytic streptococci resistant to sulphonamide. The penicillin sensitivity culture plate was so overgrown as to be useless.
6. M. Sh., female. Born 28-11-44. Premature infant-going steadily downhill from birth, jaundiced, and mildly dehydrated. Penicillin treatment coincided with clinical improvement and with gain in weight, which was rather slow at first. Total dose $-150,000$ units.

7. N. B., female. Born 18-11-44. Premature nfant with cyanotic attacks starting eleven days after birth-probably bronchopneumonia. Penicillin was given until attacks ceased, which took six days. Very ill and not weighed for some time. Total dose - 150,000 units.

Comment. Of this group five were thought to suffer from bronchopneumonia, manifested in four cases by the occurrence of cyanotic attacks about ten days after birth. In four of these cases the administration of penicillin coincided with improvement and the results were most satisfactory. In the fifth case no response took place and numerous coliform bacilli were found in the affected lung at post-mortem examination. MacGregor (1939) finds that in neonatal pneumonia the two most common and most lethal organisms are the staphylococcus aureus and organisms of the colon bacillus group. It is possible that this type of pneumonia is due to begin with to organisms sensitive to penicillin, such as the staphylococcus aureus, but that in some cases this infection cannot be overcome and a terminal invasion by bacillus coli, insensitive to penicillin, leads to a septicaemic condition. The remaining three cases were premature infants who were going steadily downhill. Here again improvement coincided with administration of penicillin, but the nature of the lesion from which they suffered unfortunately remains unknown. The amount of penicillin given depended on clinical improvement.

\section{Group (B).-Mature infants}

In the majority of the following reports the entire weight curve is shown. In a few, however, only that portion of the curve dealing with the illness described is shown. (No. 9 and 10). 
Ib. D.B.

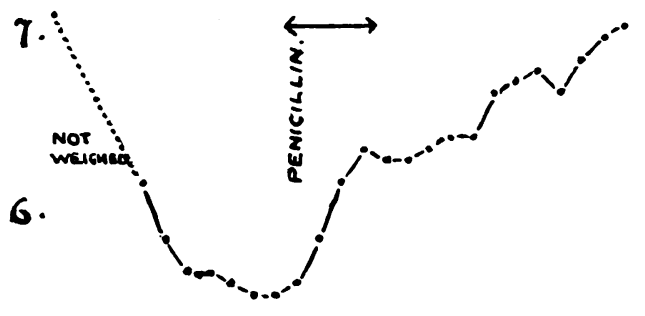

M.A.

B2.

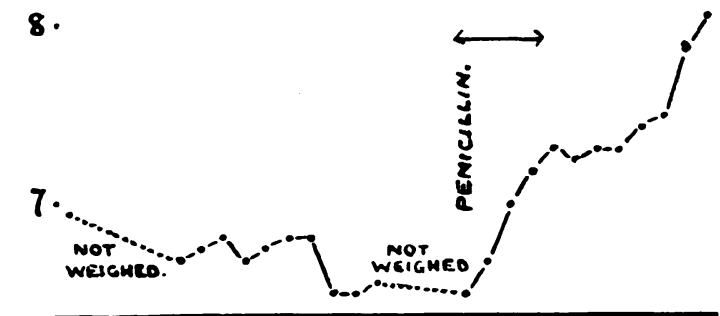

G.H.

B3.

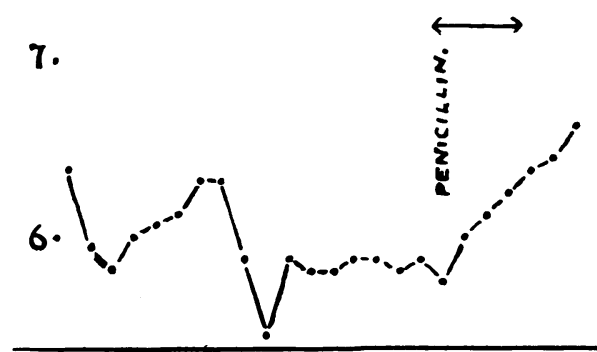

D.P.

B4.

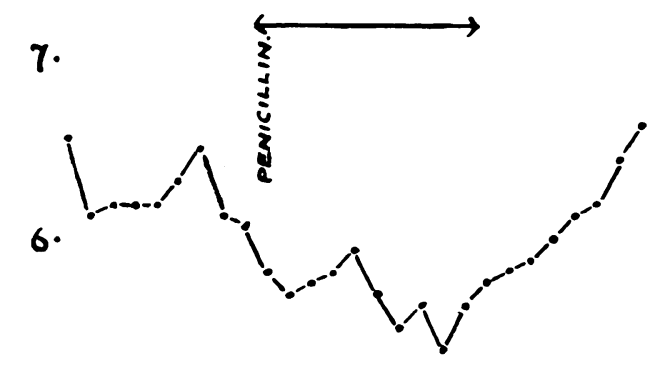

\section{MeE.}

B5
1. D. B., female. Born 16-9-44. Mother disinclined to breast feed and infant went downhill, developing bilateral acute otitis media and pharyngitis, with some dehydration. Penicillin given and ears settled clinically in four days. Weight was regained in sixteen days. Total dose-100,000 units.

2. M. A., female. Born 18-9-44. Bilateral acute otitis media. Penicillin given and breast feeding continued. Ears settled clinically in four days and birth weight was regained in two days after penicillin was begun. Total dose-100,000 units.

3. G. H., male. Born 25-9-44. Persistent vomiting due to pylorospasm treated with eumydrin. Later developed bilateral acute otitis media. Penicillin was given and breast feeding continued. Ears settled clinically in four days and birth weight was regained four days after penicillin was begun. Total dose-100,000 units.

4. D. P., male. Born 7-10-44. Bilateral acute otitis media with some dehydration. Some difficulty occurred in overcoming dehydration and reestablishing breast feeding. Ears settled clinically in three days, but penicillin was continued longer as general condition was unsatisfactory. Total dose250,000 units.

5. I. McE., female. Born 24-10-44. Jaundice and failure to gain. Developed bilateral acute otitis media. Penicillin given and breast feeding continued throughout illness. Ears settled clinically in eleven days and birth weight was regained in seven days. Total dose-200,000 units. 
lb. B.W.

B6.

9.

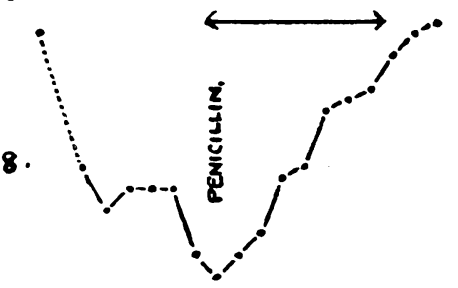

6. B. W., male. Born 24-10-44. Bilateral acute otitis media. Penicillin given and breast feeding continued. Ears settled clinically in eight days and birth weight was regained in eleven days. Total dose-200,000 units.

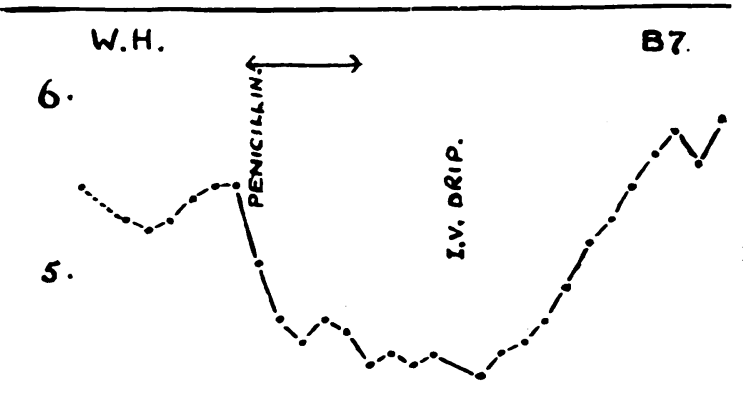

7. W. H., male. Born 25-10-44. Bilateral acute otitis media with considerable dehydration. Ears settled clinically in five days but general condition continued to deteriorate and intravenous fluids became necessary. This infant did not regain its birth weight for eighteen days. Total dose125,000 units. Slight bruising of the buttocks but no inflammation.

W.A.

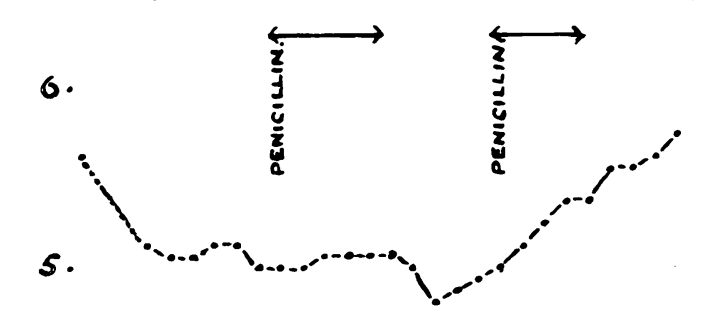

8. W. A., male. Born 29-10-44. Bilateral acute otitis media which responded well and then relapsed. Breast feeding continued during penicillin therapy. (1) Ears settled clinically in four days (125,000 units). (2) Ears settled clinically in three days $(100,000$ units). Birth weight regained in eighteen days after penicillin was begun. Total dose-225,000 units.

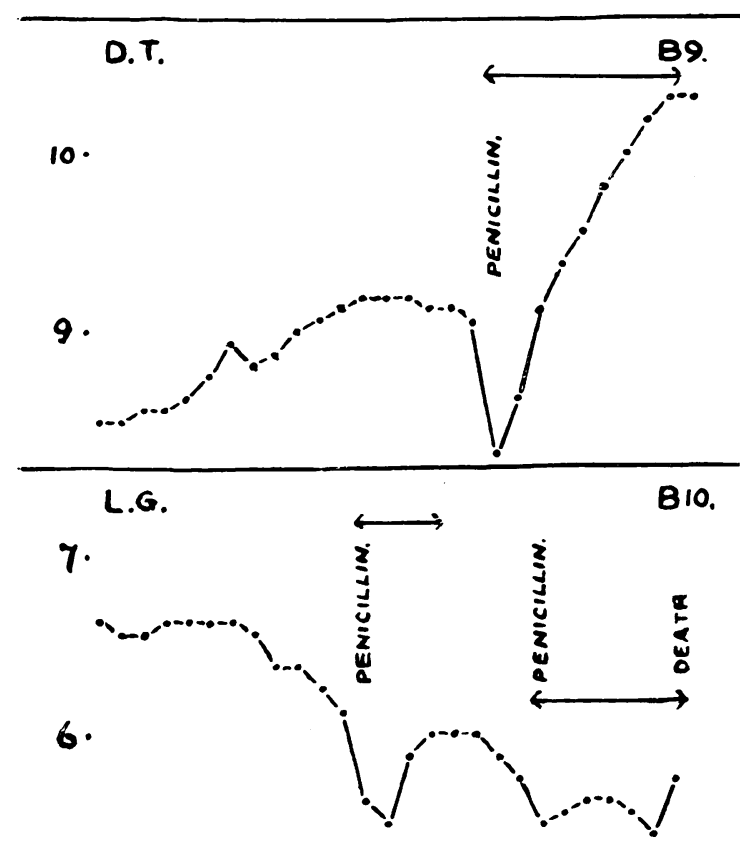

9. D. T., female. Born 26-9-44. Relapse case of bilateral acute otitis media, previously treated with sulphathiazole when ears took twelve days to settle. Penicillin given and bottle feeds continued. With penicillin ears settled clinically in nine days and weight lost was regained in four days. Total dose $-225,000$ units.

DAYS.

10. L. G., male. Born 7-10-44. Bilateral acute otitis media with dehydration. Ears settled in four days (100,000 units). Relapse occurred and on this occasion drums became normal in three days, but the infant did not improve and ultimately died with sudden elevation of temperature to $106^{\circ} \mathrm{F}$. 
Ib. R.J.
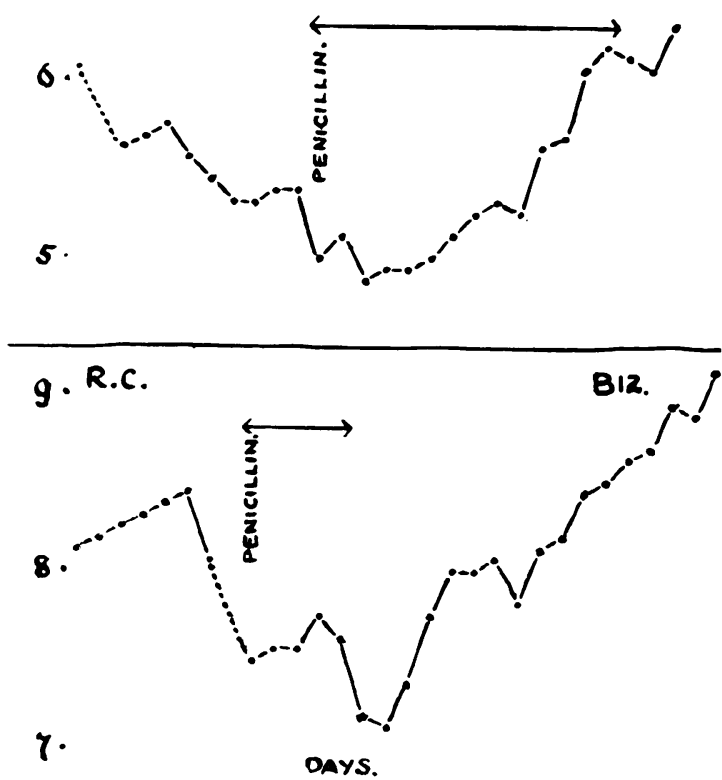

Post-mortem eXamination in Case No. 10.

Post-mortem examination (performed two hours after death) showed the presence of some muco-pus in the right middle ear. The lungs seemed normal except for a small congested area which looked macroscopically like an infarct. Other organs were normal. Direct smear from the ear showed some pus cells and culture grew only coliform bacilli. Culture from the spleen also grew many B. coli. Section of the suspicious piece of lung revealed an area of atelectasis with compensatory emphysema, haemorrhage and oedema.

Comment. A study of the case reports will show that gain in weight did not always follow administration of penicillin, but there was evidence of resolution of the inflammatory process in the middle ear in the majority of the cases and general clinical improvement. In nine of these cases of otitis media the response to penicillin was satisfactory and there was often an immediate gain in weight and improvement in the general condition. In case no. 7 the otitis media settled clinically in five days, but the general condition remained unsatisfactory and only improved after intravenous treatment. Two cases, no. 8 and 10 , relapsed. The first of these responded to the second course of penicillin, but the second case died although the inflammation appeared to have settled in three days. In those who recovered the otitis media, manifested in all cases by acutely inflamed tympanic membranes, settled clinically in three to sixteen days, average 6.5 days. The decision to continue or discontinue penicillin was made on the appearance of the tympanic membranes which were inspected daily and in two cases mistakes were made, i.e. the two cases which relapsed, no. 8 and 10. Satisfactory gain in weight did not follow the first course of
11. R. J., female. Born 7-11-44. Bilateral acute otitis media with some dehydration. Response to penicillin therapy was slow and difficulty was experienced in breast feeding necessitating complementary feeds. Ears took sixteen days to settle clinically and birth weight was regained in thirteen days. Total dose $-350,000$ units.

12. R. C., male. Born 19-9-44. Bilateral acute otitis media, dehydrated, grey and toxic looking. Penicillin was given and some difficulty occurred in reintroducing feeds. Ears settled clinically in five days and weight lost was regained in sixteen days. Total dose $-125,000$ units.

penicillin in these cases, which suggests that the inflammation had not completely subsided and that penicillin should have been continued longer. The appearance of infantile tympanic membranes is often deceptive and such errors are unavoidable. The total dose varied between 100,000 units and 350,000 units. Six cases were dehydrated in varying degree but responded well to the treatment described, only one requiring intravenous treatment. In the others, breast feeding was continued during penicillin therapy, one of the advantages of this form of treatment over the sulphonamides. The most striking features of this experiment are as follows(1) No local treatment was given or was necessary. (2) No spontaneous perforation of the tympanic membranes occurred, and (3) no myringotomies or mastoidectomies became necessary. It is interesting to compare this series with a previous series of twenty cases of otitis media treated with sulphadiazine in which only four settled with the drug alone, two drums ruptured spontaneously after the commencement of the drug, four required myringotomy and two mastoidectomy. The two groups were unfortunately not concurrent and possibly there may be some epidemiological difference between them. Nevertheless, the penicillin group seems better than the sulphadiazine group. In the case which died the post-mortem findings were not satisfactory, but it is possible that an invasion by $\mathrm{B}$. coli, such as can occur in the first month of life, caused a septicaemia which was responsible for death. The role of $\mathbf{B}$. coli in neonatal infections, particularly pneumonias, is fully discussed by MacGregor (1939).

Additional cases. A few cases which have not for various reasons been included in the series remain to be described. 
1. H. P., male. Born 28-9-44. Weight at birth, $7 \mathrm{lb}$. 13 oz. About one month after birth, during which time it had apparently been well, this infant developed nasopharyngitis with much purulent nasal discharge and signs of right upper lobe pneumonia. Nasal swab showed the presence of staphylococcus aureus, sensitive to penicillin and sulphonamides. Penicillin treatment caused the nasopharyngitis to resolve in three days, but the infant remained rather cyanosed although no clinical evidence of congenital heart disease could be found on repeated examination. This child ultimately died and post-mortem examination revealed the presence of bronchitis with an accessory right upper lobe lying in the mediastinum, congenital pulmonary stenosis, left otitis media and mastoiditis. Post-mortem swabs from mastoid and lung, when cultured, grew coliform bacilli resistant to penicillin. In all 359,375 units of penicillin were administered, but the post-mortem examination showed that, apart from the nasopharyngitis which was probably responsible for the otitis media and mastoiditis and which responded well to penicillin treatment, the case was not one likely to be benefited by such treatment.

2. A. F., male. Born 7-9-44. Weight at birth, 5 lb. 12 oz. Eight days after birth this infant developed pneumonia and was given a full course of sulphamezathine, with some improvement. A small abscess appeared on its left buttock. It then relapsed, by which time penicillin had become available. This treatment was started but the infant only survived long enough to have 25,000 units (eight doses). Post-mortem examination revealed confluent bronchopneumonia involving almost the whole of the right lung, with some patches in the left lung, left otitis media and mastoiditis and an abscess in the left buttock which had developed before the administration of penicillin. Direct smear from lung showed gram-positive cocci, few pneumococci and bacilli. Cultures gave the following mixed results: lung-coliform bacilli resistant to penicillin; mastoid-staphylococcus albus sensitive to penicillin and B. Morgan 1 resistant to penicillin; buttock abscess-staphylococcus aureus sensitive to penicillin. In this case penicillin was given too late and did not have a reasonable chance.

3. D. S., male. Born 16-11-44. Weight at birth, $5 \mathrm{lb}$. $1 \mathrm{oz}$. This was a premature infant which went gradually downhill and became mildly dehydrated eight days after birth. Five days later it developed signs of pneumonia at its right base. Penicillin treatment was begun, but it only survived long enough to have 6250 units (two doses). Postmortem examination and section of the lung confirmed the presence of bronchopneumonia. Here again treatment was begun too late.

4. H. B., male. Born 16-9-44. Weight at birth, $3 \mathrm{lb}$. $6 \mathrm{oz}$. This was a small premature infant which gradually lost weight and nine days after birth began to have cyanotic attacks. These attacks continued from time to time and seven days later penicillin was given. This child only lived long enough to have 12,000 units (four doses). Post-mortem examination revealed the presence of much bronchopneumonia affecting mainly the right lung. Direct smear from lung showed grampositive cocci and the culture grew only coliform bacilli resistant to penicillin. Penicillin treatment was begun too late in this case.

5. R. B., male. Born 30-11-44. Weight at birth, $4 \mathrm{lb} .1 \mathrm{oz}$. This was a premature infant who had a cyanotic attack five days after birth. Although rather early in onset it was decided to try penicillin, but it only received one injection before death. Post-mortem examination showed massive haemorrhages into both lungs, a large subdural haemorrhage on the right side and a tentorial tear. Pus, containing pneumococci, was also found in the right middle ear. Section from the lung showed evidence of inhalation of amniotic fluid, haemorrhage and atelectasis. This was not a suitable case for penicillin.

These cases illustrate the difficulties which beset the diagnosis in the neonatal period. They also confirm the importance of the date of onset of respiratory signs. This is well described in MacGregor's (1939) excellent article on pneumonia in the newborn.

\section{Discussion}

The results described are encouraging and it is appropriate that this should be so in infancy because Florey (1944), describing the early clinical trials of penicillin and the great difficulty in finding the adequate dosage, says 'Eventually after trial and error, a baby provided the foundation on which an adequate dose to eliminate infection was established as 1000 units per pound of body weight in 24 hours.' It will be noticed that the doses used in the present series are much larger as experience suggested that better results could be so obtained.

References to the use of penicillin in infancy are not so far numerous. Christie and Garrod (1944) mention an infant suffering from staphylococcal peritonitis and perinephric abscess, to whom a drip containing penicillin was given into the marrow. They also describe two cases of chronic otitis media where instillation of penicillin solution caused penicillin sensitive organisms to disappear from the discharge and report favourably one case of acute bilateral otitis media which was cured in seven days. Morgan, Christie and Roxburgh (1944) include three infants in their description of their experience in systemic administration of penicillin but give no details of the nature of the illnesses from which these infants suffered. One received penicillin by intramuscular injection $(28,000$ units, volume of injection $-1.5 \mathrm{c.cm}$.) and it was well tolerated. The other two had tibial transfusions (4500 and 22,500 units respectively) but the second transfusion refused to flow quickly enough to introduce an adequate amount. No changes were found in the marrow as a result of these transfusions. They suggest that drip transfusion into the bone marrow of the tibia may be of value in the administration of penicillin to infants. In a further article by the same authors dealing with the use of penicillin in skin disease, three infants are described, two suffering from impetigo and one from eczema. Two of these cases, which received local treatment with penicillin ointment, relapsed after improvement had occurred. Burns and Gunn (1944) describe five cases of gastro- 
enteritis associated with otitis media, mastoiditis and in one case pyelitis. Four cases received penicillin, two locally into mastoidectomy wounds, one intravenously and one, which developed pneumococcal meningitis, both intramuscularly and intrathecally. Two cases died but no untoward results of any of these methods of penicillin administration are reported. All these cases received sulphonamides in addition so that it is difficult to assess the part played by penicillin.

All the cases in the present series were born in hospital. They were therefore under constant observation and were rarely allowed to become grossly dehydrated. In considering the method of administration to be employed, it was decided that intramuscular drips would be difficult to control in young infants and intratibial drips have not been found entirely satisfactory. It was therefore decided to employ the intramuscular route and of the twenty cases described in only two cases did the buttocks present a somewhat bruised appearance, but no inflammation developed. This happy experience of intramuscular therapy continues. Intramuscular injection is certainly the most convenient method of administration to infants although a uniform bacteriostatic power of serum is not so obtained (Fleming et al., 1944).

One other point should be considered in assessing the results described and that is the seasonal incidence of parenteral infection. The cases described all occurred and were treated during the months of September, October, November and early December, months when these infections seem to be less severe and more easily treated. January, February,
March and April constitute the testing season for any treatment and only when these months have passed will a true estimate of the efficacy of penicillin be possible, In this connexion it is noteworthy that the continuation of this form of treatment during January, 1945, appears to show results which are not nearly as satisfactory as those reported here.

\section{Conclusion}

The encouraging results described suggest that penicillin will have a useful part to play in dealing with parenteral infection in infancy, but much further investigation is necessary to establish its exact scope.

Thanks are due to Dr. H. H. Macwilliam, Medical Superintendent, Walton Hospital, Liverpool, for permission to publish this paper and to Prof. T. B. Davie, Professor of Pathology in the University of Liverpool and Distribution Officer of the Liverpool Medical School Penicillin Committee, for much helpful advice.

\section{REFERENCES}

Alexander, M. B., and Eiser, Y. (1944). Brit. med. J., $2,425$.

Burns, M. B., and Gunn, W. (1944). Loc. cit., 178.

Christie, R. V., and Garrod, L. P. (1944). Ibid., 1, 513

Fleming, Sir A., Young. M. Y., Suchet, J., and Rowe, A. J. E. (1944). Lancet, 2, 621.

Florey, M. E. (1944). Brit. med. Bull., $2,9$.

MacGregor, A. R. (1939). Arch. Dis. Childh. 14, 323.

Morgan, H. V., Christie, R. V., and Roxburgh, I. A. (1944). Brit. med. J., 1, 515.

Roxburgh, I. A., Christie, R. V., and Roxburgh, A. C. (1944). Loc. cit., 524. 